\title{
Testing for and dating structural break in smooth time-varying cointegration parameters, with an application to retail gasoline price and crude oil price long-run relationship
}

\author{
David Neto
}

Received: 2 September 2013 / Accepted: 26 November 2014 / Published online: 7 January 2015

(C) Springer-Verlag Berlin Heidelberg 2015

\begin{abstract}
This paper outlines a methodology to test for structural break in a smooth time-varying cointegration model. We show how such a problem can be brought down to the standard procedure proposed by Hansen (J Bus Econ Stat 10:321-335, 1992). As an application, we investigate the long-run relationship between the crude oil price and the gasoline retail price for Switzerland.
\end{abstract}

Keywords Smooth time-varying cointegration - Structural break · FMLS .

Score test · FM Wald test - FMLS-based CUSUM test - Crude oil price and retail price of gasoline

JEL Classification $\quad \mathrm{C} 12 \cdot \mathrm{C} 18 \cdot \mathrm{Q} 43$

\section{Introduction}

The main appeal of cointegration theory is to model long-run relationships and to provide an economic interpretation to specifications which deal with non-stationary variables. However, many empirical investigations fail to validate cointegration relationships representing many important economic laws. This problem is frequently attributed to misspecification of the models. For example, it is well known that institutional constraints or existence of transaction costs in financial asset markets make linear specifications too restrictive, for instance, when monetary authorities use a tar-

The author was a Post-Doctoral Research Assistant at the Department of Economics, University of Geneva, when an early version of this paper was written.

D. Neto $(\bowtie)$

University of Geneva, Geneva, Switzerland

e-mail: david.neto@unige.ch 
get zone device to apply an exchange rate policy or when a price control is established by a government in a specific market.

Therefore, including nonlinearities in cointegrated models has become of great importance in these last two decades. For instance, threshold cointegration models have attracted significant attention due to its flexibility and ability to capture many features as asymmetric adjustments to long-run equilibria and/or persistence in the meanreverting effect (see Balke and Fomby 1997; Enders and Siklos 2001; Hansen and Seo 2002; Gonzalo and Pitarakis 2006; Seo 2006; Krishnakumar and Neto 2011, 2012, among others). Another important source of misspecification in cointegration models is the parameter instability. An early and popular route to face to this inconstancy consists of including structural breaks in the dynamics of interest which allow the parameters to change with respect to some specific periods (Gregory and Hansen 1996). However, this approach suffers from several drawbacks. First, it is difficult to consider many breaks regarding the sample size which can lead to a lack of power of the inference. Second, the method often detects too many significant breaks around the transition periods. Combined together, the interpretations for significant breaks are not always obvious for practitioners. If this approach seems to be appropriate to describe some economic phenomenon, (such as structural policy reforms, economic shocks, tax introduction), it may not be for long-run economic relationships for which potential changes occur slowly and smoothly over time. Therefore, smooth timevarying parameter models seem to be a promising alternative when (i) parameters exhibit a great instability and (ii) when the changes do not occur as abrupt changes but rather slowly and smoothly.

Since the early published paper of Park and Hahn (1999, henceforth PH), only few theoretical papers have investigated time-varying cointegration; one can cite Xiao (2009) and Bierens and Martins (2010, henceforth BM), and even less in the empirical literature. However, if PH and BM's methodologies are quite similar, since they both specify the time-varying cointegrating parameter as a deterministic function of time (fourier transforms for PH and Chebyshev time polynomials for BM), Xiao's methodology belongs more to the stochastic cointegration literature (despite the terminology functional coefficient used by the author to design his specification). So far, these latter models have gained less popularity in practical domain than the route proposed by $\mathrm{PH}$ and BM.

Hence, smooth time-varying cointegration models are intended to capture slow modifications of the economic environment in the long-run without abrupt changes. For instance, Park and Zaho (2010) and more recently Neto (2012) have stressed how restrictive the time-invariant cointegration assumption for the elasticities of gasoline demand could be.

However, since cointegration theory is particularly appropriate to describe longrun dynamics, it cannot be excluded that structural breaks occur over the considered period, even though the smooth and slow time-varying long-run relationship is taken into account in the model. Therefore, smooth time-varying cointegration needs to be combined with structural breaks. Therefore, this paper aims to test for structural break in a smooth time-varying cointegration model. For this purpose, we show how such a model can be brought down to a standard specification allowing us to apply Hansen's procedure (1992). We follow BM using Chebyshev time polynomials which 
are cosine functions of time and special case of the Gegenbauer polynomials. These polynomials seem to be particularly attractive for regression modelling since they are (i) orthogonal, which prevent any collinearity in the regressors, and (ii) they are able to approximate well strong nonlinear features with a modest number of Chebyshev polynomials (Bierens 1997).

The remainder of the paper is organized as follows. Section 2 presents the smooth time-varying cointegration model with structural break. Section 3 outlines the testing procedure for structural break under time-varying cointegration. Section 4 is devoted to Monte Carlo experiments: sample size in finite sample and power of the test are explored. In Sect. 5, we present an empirical application of our procedure to the long-run relationship between the crude oil price and the gasoline retail price for Switzerland from 1973 to 2012. Throughout this empirical application, we also discuss the estimation of the break date. Section 6 concludes.

\section{Smooth time-varying cointegration with structural break}

\subsection{The econometric model}

Let us consider the following model:

$$
y_{t}=\theta(t)^{\prime} x_{t}+u_{t}, \quad \Delta x_{t}=e_{t}, \quad t=1, \ldots, T,
$$

where $x_{t}$ is a $(p-1)$ vector of integrated processes of order 1 , denoted $\mathrm{I}(1), v_{t}=$ $\left(u_{t}, e_{t}^{\prime}\right)^{\prime}$ is $\mathrm{I}(0) \mathrm{p}$-dimensional process with mean $\mathbf{0}$, possibly correlated, and satisfies the functional central limit theorem. Hence, $y_{t}$ and $x_{t}$ are cointegrated and $\theta(t)^{\prime}=$ $\left(\theta_{1}(t), \theta_{2}(t), \ldots, \theta_{p-1}(t)\right)$ is the $(p-1)$ cointegration vector which is assumed to vary smoothly over time. Many specifications have been proposed for the function $\theta(t)$, one can cite, for example, PH (1999) and more recently Bierens and Martins (2010). Throughout the paper, we consider the BM's specification to describe $\theta(t)$ which consists of using Chebyshev time polynomials. These polynomials are particularly appealing since (i) they are bounded, which is required to obtain finite variances for the time-varying parameter estimator, (ii) they are orthogonal, which prevents collinearity in the regressors (that is, not the case for regular time polynomials), (iii) they are able to capture strong nonlinear characteristics with a low degree of polynomials (Bierens (1997)), which is particularly suitable for cointegrating relationships since only few variables are commonly involved in the long-run equation, and (iv) they are able to provide a good approximation of cyclical behaviours without Runge's phenomenon (see Fig. 1).

The time-varying long-run parameters are given as follows:

$$
\theta_{k}(t)=\sum_{i=0}^{T-1} \xi_{k, i, T} G_{i, T}(t),
$$

for $k=1, \ldots,(p-1)$ and where $\xi_{k, i, T}$ are Fourier coefficients uniquely defined by 
(a)

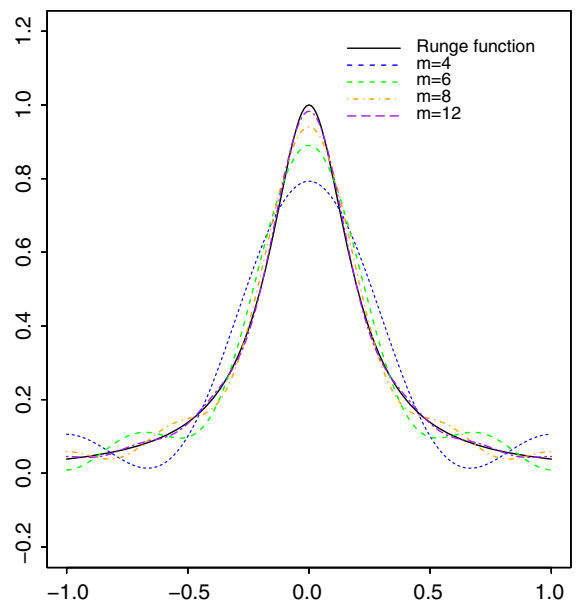

(b)

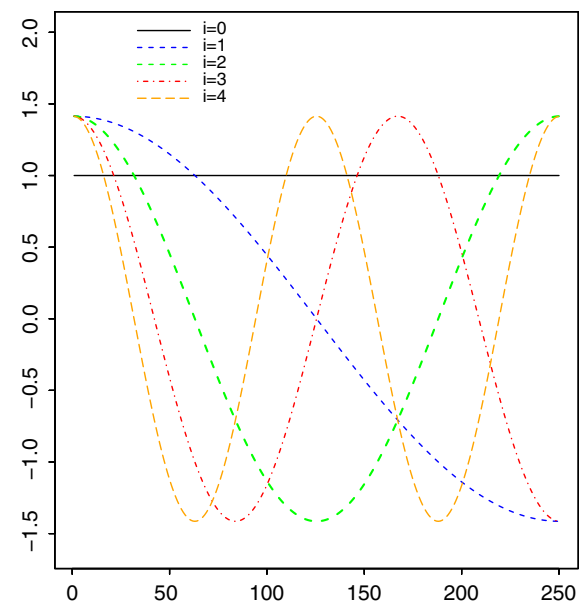

Fig. 1 a Approximation of the Runge function using Chebyshev time polynomial regression. b Chebyshev time polynomials

$$
\xi_{k, i, T}=T^{-1} \sum_{t=1}^{T} \theta_{k}(t) G_{i, T}(t),
$$

where $G_{i, T}(t)$ are the Chebyshev time polynomials defined as:

$$
G_{i, T}(t)= \begin{cases}1, & \text { for } i=0, \\ \sqrt{2} \cos \left(T^{-1} i \pi(t-0.5)\right), & \text { for } i>0,\end{cases}
$$

for $t=1, \ldots, T$. Note that the dynamic described by (1)-(3) is allowed by the orthonormality property of the Chebyshev polynomials, i.e. $\forall(i, j) \in \mathbb{Z}$, we have $T^{-1} \sum_{t=1}^{T} G_{i, T}(t) G_{j, T}(t)=\mathbb{I}_{(i=j)}$. If we assume that the trend function $\theta$ is reasonably smooth, a Fourier approximation can be used to approximate quite well $\theta(t)$ with a modest number of Chebyshev polynomials:

$$
\theta_{k, m}(t)=\sum_{i=0}^{m} \xi_{k, i, T} G_{i, T}(t)
$$

for some fixed $m \ll T-1 .^{1}$ Thus, we can see that $m=0$ corresponds to the timeinvariant cointegration context. For simplicity, we use $\theta(t)$ to denote the truncation $\theta_{m}(t)$.

To combine the occurrence of a structural break and time-varying long-run parameters, we assume that the degree of smoothness of the long-run parameter dynamic is not affected by the structural change, which means that $m$ in (4) is the same before and

\footnotetext{
1 See Bierens (1997) for technical details on Chebyshev time polynomials.
} 
after the change. Only the value of the Fourier coefficients $\xi_{k, i, T}$ are affected by the break. We believe this assumption acceptable to describe the economy in the long run.

Let us now modify the model (1) to incorporate a structural break in the smooth dynamic of $\theta(t)$; thus, we have:

$$
y_{t}=\theta(t)^{\prime} x_{t}+\theta_{c}(t)^{\prime} x_{t} \delta_{t}+u_{t}
$$

where $\delta_{t}=1$ if $t>\left[T \tau_{o}\right], 0$ otherwise, [·] stands for "integer part", $\tau_{o}$ is the (constant) break fraction such as $\tau_{o} \in\left[\tau_{L}, \tau_{U}\right] \subset(0,1)$, and $\theta_{c}(t)=\sum_{i=0}^{m} \xi_{c, k, i, T} G_{i, T}(t)$, with $\xi_{c, k, i, T} \neq \xi_{k, i, T}$.

In order to build a test for structural break from (5), it is convenient to rewrite the previous model by factorizing the Fourier coefficients $\xi_{i, T}=\left(\xi_{1, i}, \ldots, \xi_{k, i}, \ldots \xi_{(p-1), i}\right)^{\prime}$ and $\xi_{c, i, T}=\left(\xi_{c, 1, i}, \ldots, \xi_{c, k, i}, \ldots \xi_{c,(p-1), i}\right)^{\prime}$

$$
y_{t}=\sum_{i=0}^{m} \xi_{i}^{\prime} G_{i, T}(t) x_{t}+\sum_{i=0}^{m} \xi_{c, i}^{\prime} G_{i, T}(t) x_{t} \delta_{t}+u_{t} .
$$

The matrix form of (6) is given by

$$
Y=\mathbf{Z v e c}(\Xi)+U
$$

where $\mathbf{Z}=\left(Z_{1}, \ldots, Z_{(p-1)}, Z_{c, 1}, \ldots, Z_{c,(p-1)}\right)$ is a $(T \times 2(p-1)(m+1))$ matrix, $Z_{k}=\mathbf{G}^{(m)} \odot X_{k}$, and $Z_{k}=\mathbf{G}^{(m)} \odot X_{c, k}$, with $\odot$ denotes the Hadamard product, $\mathbf{G}^{(m)}$ a $(T \times(m+1))$ matrix such that $\mathbf{G}^{(m)}=\left(G_{0, T}, \ldots, G_{m, T}\right)$, and where the terms $G_{i, T}, Y, X_{k}, X_{c, k}$, and $U$, stack over time the elements $G_{i, T}(t), y_{t}, x_{k, t}, x_{k, t} \delta_{t}$, and $u_{t}$, respectively, for $k=1, \ldots, p-1$, and $\Xi=\left(\xi, \xi_{\mathbf{c}}\right)$ is $(m+1) \times 2(p-1)$, where

$$
\xi=\left(\begin{array}{cccc}
\xi_{10} & \xi_{20} & \ldots & \xi_{(p-1) 0} \\
\xi_{11} & \xi_{21} & \ldots & \xi_{(p-1) 1} \\
\vdots & \vdots & & \vdots \\
\xi_{1 m} & \xi_{2 m} & \ldots & \xi_{(p-1) m}
\end{array}\right) \in \mathbb{R}^{m+1} \times \mathbb{R}^{p-1}
$$

and $\xi_{c}$ defined similarly.

Note that $Z_{k}$ and $Z_{c, k}$ remain $I(1)$ if $X_{k}$ is $I(1)$. Indeed, let us set $x_{h, t=0}=0$ and $e_{k, t}$ in (1) being 0 mean with variance $\sigma$, we have for $m=0, G_{0, T} X_{k}=X_{k}$ and for $m>0, z_{k, t}=G_{m, T-1}(t) e_{k, t}+\left(G_{m, T-1}(t)-G_{m, T-1}(t-1)\right) \sum_{j=1}^{t-1} e_{k, t-j}$. It is straightforward to see that the variance of $z_{k, t}$ tends to infinity as $T \rightarrow \infty$. The argument is similar for $Z_{c, k}$.

2.2 The fully modified least squares estimator and fully modified Wald statistic for testing time-invariant cointegration hypothesis

It is well known that the performance of the estimator of cointegrating vectors based on the OLS is affected by the existence of second-order (or endogeneity) biases (see 
Park and Phillips 1988; Phillips and Hansen 1990). Whilst these biases do not affect the consistency of the estimator, which is super-consistent, its asymptotic distribution remains not standard. Several efficient approaches have been proposed in order to obtain median-unbiased and asymptotically normal estimates, property which is required for our test. Among of those, we use the widespread fully modified least squares (FMLS) estimator proposed by Phillips and Hansen (1990) which consists of a nonparametric correction of the single-equation estimates. Considering a possible correlation between $e_{t}$ and $u_{t}$ in (1), the FMLS can be applied to (7) in order to take into the account the endogeneity bias. Let us partition conformably with $v_{t}$ its long-run covariance matrix $\Omega$ as follows

$$
\Omega=\left(\begin{array}{cc}
\omega_{u u} & \omega_{e u}^{\prime} \\
\omega_{e u} & \Omega_{e e}
\end{array}\right)=2 \pi f_{v v}(0)
$$

where $f_{v v}(\lambda)$ is the spectral density of $v_{t}$; the FMLS estimator of $\Xi$ is

$$
\operatorname{vec}\left(\hat{\Xi}^{+}\right)=\left(\mathbf{Z}^{\prime} \mathbf{Z}\right)^{-1}\left(\mathbf{Z}^{\prime} Y^{+}-T \hat{\Gamma}^{+}\right)
$$

where $Y^{+}(T \times 1)$ is the observation vector for $y_{t}^{+}=y_{t}-\hat{\omega}_{u e}^{\prime} \hat{\Omega}_{e e}^{-1} \Delta x_{t}$ and where $\hat{\Gamma}^{+}=\hat{\Lambda}\left(\begin{array}{c}1 \\ -\hat{\Omega}_{e e}^{-1} \hat{\omega}_{e u}\end{array}\right)$ represents the estimated bias due to the endogeneity with $\hat{\Omega}_{e e}, \hat{\omega}_{e u}$ and $\hat{\Lambda}$ consistent estimates of the corresponding elements of $\Omega$, and of $\Lambda=$ $\sum_{j=0}^{\infty} \mathbb{E}\left(e_{0} v_{j}^{\prime}\right)$, respectively. The FM estimator of the variances for individual elements $\operatorname{vec}(\hat{\Xi})_{l}^{+}$of $\operatorname{vec}\left(\hat{\Xi}^{+}\right)$is denoted $\hat{\mathbb{V}}^{+}\left(\xi_{k i}\right)$ and $\hat{\mathbb{V}}^{+}\left(\xi_{c, k i}\right)$ for which the expression is given by $\hat{\omega}_{\text {uu.e }}\left[\left(\mathbf{Z}^{\prime} \mathbf{Z}\right)^{-1}\right]_{l l}$, where $l l$ is the diagonal index of the matrix $\hat{\mathbb{V}}^{+}(\operatorname{vec}(\boldsymbol{\Xi}))$ corresponding to the index $k i$ of the individual Fourier parameter, and where $\hat{\omega}_{u u \cdot e}=$ $\hat{\omega}_{u u}-\hat{\omega}_{e u}^{\prime} \hat{\Omega}_{e e}^{-1} \hat{\omega}_{e u}$.

Since the FMLS estimator is asymptotically normally distributed and noting that for all $t$ and $\forall(m, T)$ we have $\sum_{i=0}^{m} G_{i, T}^{2}(t)<2(m+1)$, which insures the FM variance of the time-varying long-run parameters to be finite, we have the following fully modified variance estimators: $\hat{\mathbb{V}}^{+}\left(\theta_{k}(t)\right)=\sum_{i=0}^{m} G_{i, T}^{2}(t) \hat{\mathbb{V}}^{+}\left(\xi_{k i}\right)$ and $\hat{\mathbb{V}}^{+}\left(\theta_{c, k}(t)\right)=$ $\sum_{i=0}^{m} G_{i, T}^{2}(t) \hat{\mathbb{V}}^{+}\left(\xi_{c, k i}\right)$.

Hence, it is possible to conduct inference directly on the time-varying cointegrating vectors rather than on the Fourier coefficients $\xi_{i}$ (see Neto 2012). However, inference on Fourier coefficients can be useful if we want to test the time-invariant cointegration hypothesis since $m=0$ corresponds to the time-invariant dynamic. Therefore, such a test merely consists of testing the null hypothesis under which all the components of the matrix $\Xi$ are 0 except for the first row. This test can be performed using a fully modified (FM) Wald statistic which behaves as a Chi squared with 2( $p-1)$ degrees of freedom (see BM 2010, and Neto 2012). 


\subsection{Selection of $m$}

The selection procedure for the order $m$ is not clearly described in the literature. Bierens (1997) and BM (2010) suggest that information criteria such as the HannanQuinn criterion (HQC), the Schwarz criterion, or the Bayesian information criterion (BIC) should be consistent for $m<\infty .^{2}$ Recall that the difference between these three criteria resides on the penalty term. Thus, only the number of parameters in the model appears in the AIC, whereas the BIC also penalizes by the sample size. In our empirical application, we will prefer the HQC for which the growth of the penalty is slower than the two latter ones (it depends on $\log \log T$ ). However, if our series cointegrate together, the inference from the FMLS will be standard and a Student's test of significance of each Chebyshev polynomials in the regression should also be valid to select $m$. An alternative approach, but somewhat computationally heavier, would be to proceed by cross-validation.

However, no matter what the retained selection procedure is, a general degree of $m$ has to be chosen, and this choice remains ad hoc. Indeed, according to the empirical issue that the practitioner is coping with, a maximum source of time variation, in line with the theory, with the economic beliefs or with the existing literature, will be preferred.

\section{Testing for structural break in a smooth cointegrated relationship}

We are interested in testing the null hypothesis that states the stability of the parameters:

$$
\mathrm{H}_{0}: \xi_{c}=\mathbf{0}, \quad \text { or } \mathbf{R} \operatorname{vec}(\Xi)=\mathbf{0}
$$

where $\mathbf{R}$ is a $(m+1)(p-1) \times 2(m+1)(p-1)$ selection matrix. When the change point $\left[T \tau_{o}\right]$ is known, the Lagrange multiplier $(L M)$ statistic to test $\mathrm{H}_{0}$ is built from the partial sum process of sample scores $S(\tau, \mathbf{R})=\mathbf{R} \sum_{t=1}^{[T \tau]} S_{t}^{+}$, where $S_{t}^{+}$is the scores of the FM problem (see Hansen 1992 for details), the LM statistic is given by:

$$
L M_{T}(\tau, \mathbf{R})=\operatorname{tr}\left\{S(\tau, \mathbf{R})^{\prime}\left(\hat{\omega}_{u u \cdot e} \mathbf{V}(\tau, \mathbf{R})\right)^{-1} S(\tau, \mathbf{R})\right\}
$$

where $\mathbf{V}(\tau, \mathbf{R})=\mathbf{R}\left[M_{[T \tau]}-M_{[T \tau]} M_{T T}^{-1} M_{[T \tau]}\right] \mathbf{R}^{\prime}$ and $M_{[T \tau]}$ is the subsample empirical cross-product matrix, $M_{[T \tau]}=\sum_{t=1}^{[T \tau]} x_{t} x_{t}^{\prime} .^{3}$

Since the FMLS estimator is asymptotically normally distributed, Hansen (1992) showed that the statistic $L M_{T}$ behaves as a $(m+1)(p-1)$ degrees of freedom Chi-squared distribution.

When the true break point $\left[T \tau_{o}\right]$ is unknown, the $L M$ test is performed at each possible change point $[T \tau]$ where $\tau$ should be set in the interior of $(0,1)$. Indeed,

\footnotetext{
2 Notice that the authors do not provide a formal proof of this consistency.

${ }^{3}$ If equation (1) includes an intercept, this matrix should be $M_{[T \tau]}=\sum_{t=1}^{[T \tau]}\left(x_{t}-\bar{x}\right)\left(x_{t}-\bar{x}\right)^{\prime}$.
} 
Table 1 Asymptotic critical values for the sup $L M$ statistic

\begin{tabular}{|c|c|c|c|c|c|c|c|c|c|}
\hline \multirow[b]{2}{*}[\tau_{L},1-\tau_{L}]{} & $90 \%$ & $95 \%$ & $99 \%$ & $90 \%$ & $95 \%$ & $99 \%$ & $90 \%$ & $95 \%$ & $99 \%$ \\
\hline & \multicolumn{3}{|c|}{$(m=1, p=2)$} & \multicolumn{3}{|c|}{$(m=2, p=2)$} & \multicolumn{3}{|c|}{$(m=3, p=2)$} \\
\hline$[0.15,0.85]$ & 11.7 & 13.4 & 17.2 & 14.8 & 16.5 & 19.8 & 17.2 & 19.0 & 22.6 \\
\hline$[0.10,0.90]$ & 12.3 & 13.9 & 17.7 & 15.1 & 17.0 & 20.3 & 17.5 & 19.2 & 22.9 \\
\hline \multirow[t]{2}{*}[0.05,0.95]{} & 12.9 & 14.5 & 18.2 & 15.8 & 17.5 & 21.1 & 17.5 & 19.3 & 22.9 \\
\hline & \multicolumn{3}{|c|}{$(m=4, p=2)$} & \multicolumn{3}{|c|}{$(m=5, p=2)$} & \multicolumn{3}{|c|}{$(m=6, p=2)$} \\
\hline$[0.15,0.85]$ & 19.2 & 21.0 & 25.2 & 21.3 & 24.2 & 50.0 & 23.1 & 27.7 & 91.5 \\
\hline$[0.10,0.90]$ & 19.4 & 21.4 & 26.7 & 21.3 & 24.2 & 50.0 & 23.1 & 27.7 & 91.5 \\
\hline \multirow[t]{2}{*}[0.05,0.95]{} & 19.4 & 21.4 & 26.7 & 21.3 & 24.2 & 50.0 & 23.1 & 27.7 & 91.5 \\
\hline & \multicolumn{3}{|c|}{$(m=1, p=3)$} & \multicolumn{3}{|c|}{$(m=2, p=3)$} & \multicolumn{3}{|c|}{$(m=3, p=3)$} \\
\hline$[0.15,0.85]$ & 16.3 & 18.1 & 22.2 & 20.6 & 22.7 & 27.4 & 24.3 & 26.4 & 31.0 \\
\hline$[0.10,0.90]$ & 16.8 & 18.6 & 22.5 & 21.1 & 23.1 & 27.7 & 24.4 & 26.5 & 31.0 \\
\hline \multirow[t]{2}{*}[0.05,0.95]{} & 17.4 & 19.2 & 23.1 & 21.3 & 23.3 & 27.9 & 24.4 & 26.5 & 31.0 \\
\hline & \multicolumn{3}{|c|}{$(m=4, p=3)$} & \multicolumn{3}{|c|}{$(m=5, p=3)$} & \multicolumn{3}{|c|}{$(m=6, p=3)$} \\
\hline$[0.15,0.85]$ & 26.9 & 29.2 & 34.4 & 29.4 & 32.1 & 38.7 & 29.8 & 32.7 & 38.2 \\
\hline$[0.10,0.90]$ & 26.9 & 29.2 & 34.4 & 29.4 & 32.1 & 38.7 & 29.8 & 32.7 & 38.2 \\
\hline$[0.05,0.95]$ & 26.9 & 29.2 & 34.4 & 29.4 & 32.1 & 38.7 & 29.8 & 32.7 & 38.2 \\
\hline
\end{tabular}

Calculated from 10,000 replications and with a sample size 1,000

Hansen (1992) discussed the inconsistency of the test when one uses $\tau=0$. Thus, for $\tau \in\left[\tau_{L}, \tau_{U}\right] \subset(0,1)$, the supremum $L M$ statistic can be used to test $\mathrm{H}_{0}{ }^{4}$

$$
\sup L M(\mathbf{R})=\sup _{\tau \in\left[\tau_{L}, \tau_{U}\right]} L M_{T}(\tau, \mathbf{R})
$$

with

$$
\sup L M(\mathbf{R}) \rightarrow_{d} \sup _{\tau \in\left[\tau_{L}, \tau_{U}\right]} L M(\tau)
$$

where " $\rightarrow d$ " denotes the convergence in distribution (Hansen 1992).

The choice for $\tau_{L}$ and $\tau_{U}$ is known to be somewhat tricky. Even though a consensus seems to emerge from the empirical literature, suggesting $\tau_{L}=1-\tau_{U}=0.15$, other values, such as 0.10 or 0.05 , can also be tried in practice. For that reason, Table 1 provides critical values of the sup $L M$ statistic for these latter values, for $m=(1,2,3,4,5,6)$ and $p=(2,3)$. These limiting distributions were obtained by Monte Carlo simulations using a sample size 1,000 and 10,000 replications.

\footnotetext{
4 Notice that the supremum is also favored here to minimize the consequences of the end-point problem, inherent to polynomial regression technics.
} 
Table 2 Rejection frequencies of the sup $L M$ statistic under $\mathrm{H}_{0}$ with $5 \%$ level

Calculated from 5,000 replications

\begin{tabular}{lll}
\hline $\mathrm{T}$ & $m=1$ & $m=2$ \\
\hline 100 & 0.030 & 0.023 \\
250 & 0.040 & 0.031 \\
500 & 0.048 & 0.041 \\
1,000 & 0.052 & 0.049 \\
\hline
\end{tabular}

Note that other statistics are available to test $\mathrm{H}_{0}$ when the break timing is unknown, like the mean $L M$ test. However, we only present the Supremum statistic since the use of the others is immediate (see Hansen 1992).

\section{Monte Carlo experiments}

Throughout the experiments, we have considered a two-dimensional time-varying cointegrating system according to the following data generating process (DGP):

$$
y_{t}=\theta(t) x_{t}+\theta_{c}(t) x_{t} \delta_{t}+u_{t}, \quad x_{t}=x_{t-1}+e_{t},
$$

generated from

$$
v_{t}=\left(e_{t}, u_{t}\right) \sim \operatorname{iid} N\left(\left(\begin{array}{l}
0 \\
0
\end{array}\right),\left(\begin{array}{cc}
1 & 0.4 \\
0.4 & 1
\end{array}\right)\right)
$$

with $m=1$, 2, i.e. $\theta(t)=\xi_{0}+\sum_{i=1}^{m} \xi_{i} G_{i, T}(t)$ and $\theta_{c}(t)=\xi_{c, 0}+\sum_{i=1}^{m} \xi_{c, i} G_{i, T}(t)$. Our following simulations consist of 5,000 Monte Carlo replications.

\subsection{Finite sample performance}

In order to investigate finite sample properties of the sup $L M$ test, we simulate the DGP (11) for $T=100,250,500,1000$, with the following parameter values: $\xi_{c, i}=0$ (for $i=\{0,1\}$ ), $\xi_{0}=1.5$ and $\xi_{1}=0.2$ if $m=1 ; \xi_{c, i}=0$ (for $i=\{0,1,2\}$ ), $\xi_{0}=1.5, \xi_{1}=-1.2$ and $\xi_{2}=0.2$, when $m=2$. Table 2 reports rejection frequencies of the sup $L M$ test statistic at $5 \%$ significance level for $\tau_{L}=0.15$. Our results show that despite a slight size distortion at this significance level, the sup $L M$ test performs reasonably well overall. Note that these results are independent of the calibration.

\subsection{Power}

We examine the power of the test under the alternative [i.e. with $\xi_{c, i} \neq 0$ in (11)], ${ }^{5}$ by considering two scenarios depending on the location of the break. In the first one, we

\footnotetext{
5 The values used for our experiments are the following: $\xi=(1.5,0.2)^{\prime}$ and $\xi_{c}=(0.2,-0.7)^{\prime}$ for $m=1 ; \xi=(1.5,-1.2,0.2)^{\prime}$ and $\xi_{c}=(-0.7,-0.1,0.2)^{\prime}$ for $m=2$.
} 
Table 3 Rejection frequencies of the sup $L M$ statistic under $\mathrm{H}_{1}$ with $5 \%$ level when the break occurs in the middle and at the beginning of the sample

replications

\begin{tabular}{lll}
\hline $\mathrm{T}$ & $m=1$ & $m=2$ \\
\hline & & $\tau_{o}=0.5$ \\
100 & 1.00 & 0.944 \\
250 & 1.00 & 1.00 \\
500 & 1.00 & 1.00 \\
& & $\tau_{o}=0.1$ \\
100 & 0.607 & 0.668 \\
250 & 0.977 & 0.994 \\
500 & 1.00 & 1.00 \\
\hline
\end{tabular}

set a break which occurs in the middle of the sample, that is, $\tau_{o}=0.5$, whereas in the second situation, the break occurs at the beginning of the sample, that is, $\tau_{o}=0.1$.

Table 3 presents the rejection frequencies of the sup $L M$ statistic under the alternative with 5\% level. As expected, Table 3 suggests that the power is affected by the location of the break for small sample sizes $(T=100)$ but not for moderate and large sample sizes $(T=250,500)$.

\section{Empirical application}

Our empirical investigation uses the quarterly crude oil prices (in Swiss Francs) and the Swiss gasoline retail real prices from Q1.1973 to Q4.2012. These quarterly time series were extracted from DATASTREAM database and from the Swiss Federal Office of Statistics. Figure 2a depicts the evolution of both price series which clearly exhibit two sharp falls. The first one, at the end of 1985, which corresponds to the oil aftershock, and a recent one at the end of the sample in August 2008 when the oil price collapsed following the "third oil shock". 6

Since the first oil shock in 1973, the environmental preoccupations kept growing from politics minds as well as from the consumers and slowly led to preference shifts in demand of energy. Therefore, the response of the retail gasoline price to the crude oil price has changed substantially in these last decades. Even though the sharp fall in 1985 was mainly caused by new oil exporters on the market such as Mexico, Alaska, or North Sea, it is also a consequence of the new growing energy economic environment driven by energy savings. Therefore, a smooth time-varying cointegration model seems to be appropriate to describe the long-run dynamic between these two variables. Moreover, we can clearly distinguish two regions in the scatter plot 2(b): one before 1985 and one after 1985. On the contrary, the break observed in Q3.2008 does not turn up as strongly as the previous one. Notice that this collapse occurs in the trimmed region required by the sup $L M$ test procedure ( $15 \%$ was chosen for this empirical study), and consequently, it will not be considered as a possible break point by the procedure.

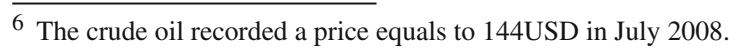




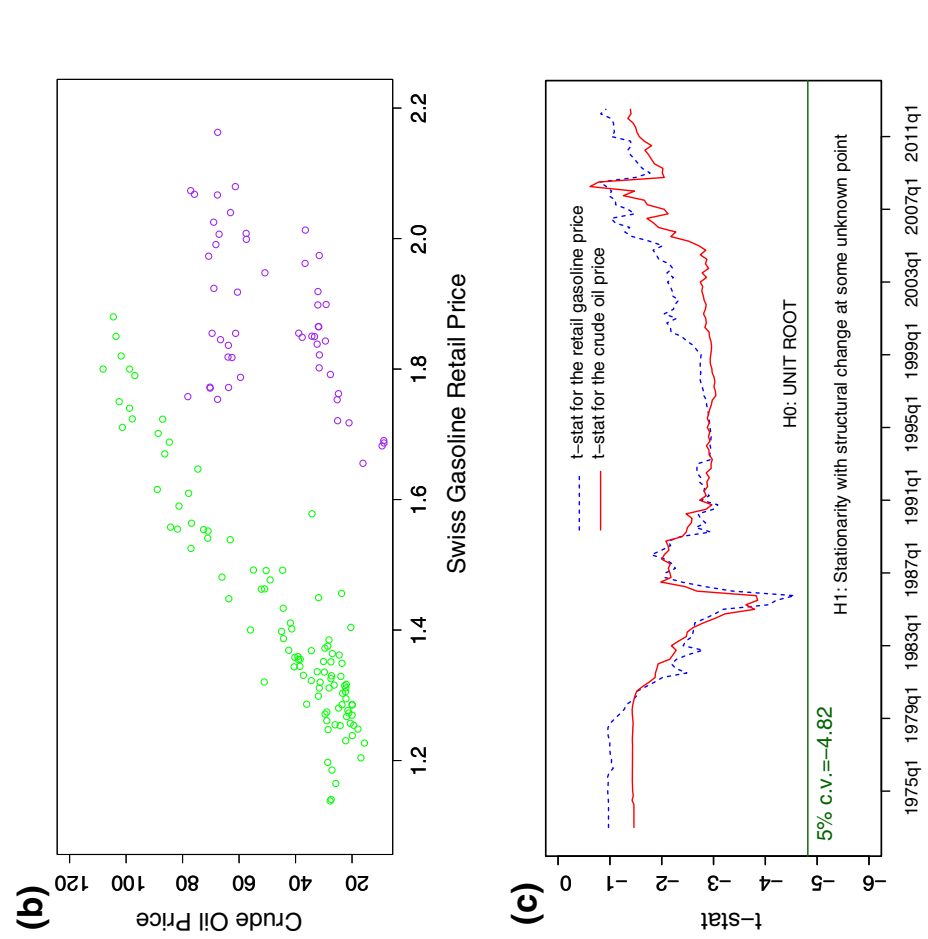

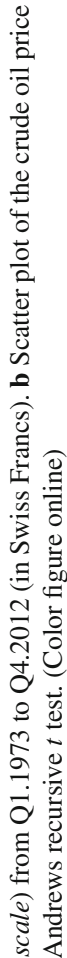

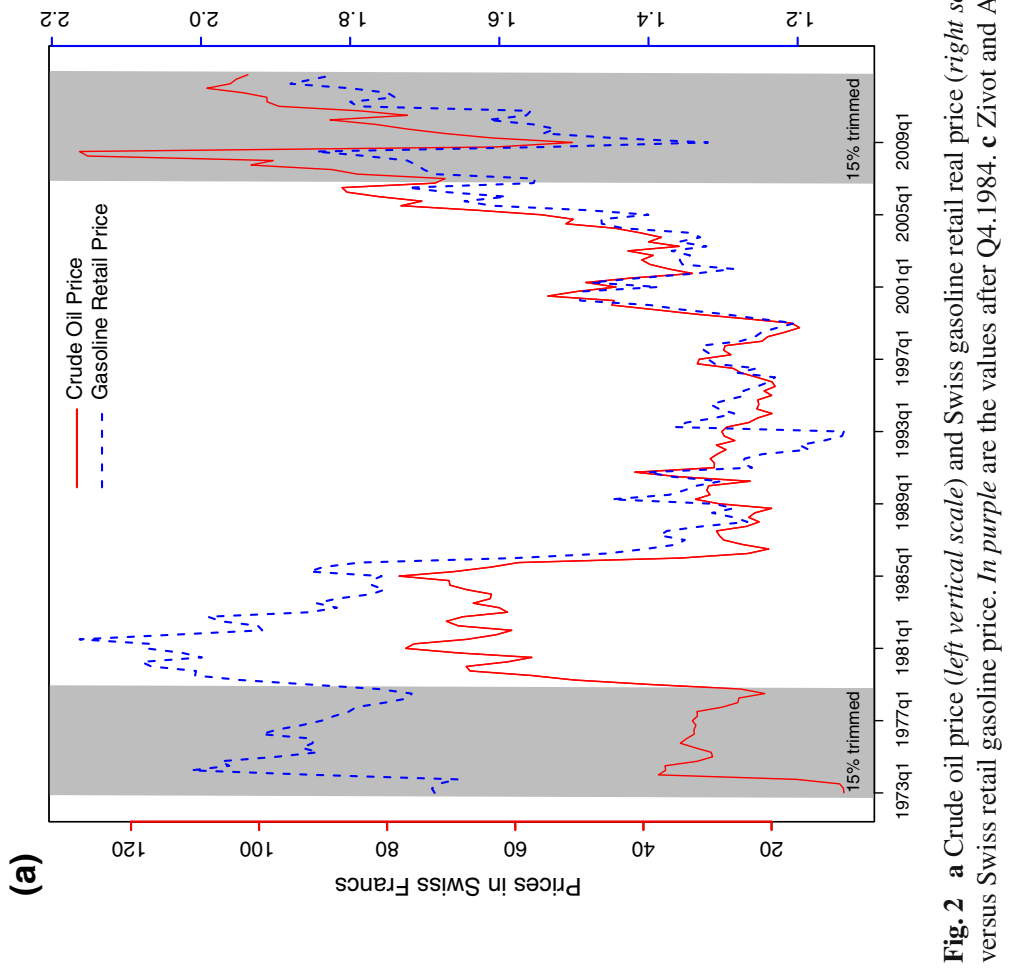




\subsection{Unit root properties}

Since we suspect a structural change in the series, standard unit root tests would lead to spurious acceptance. A test able to statistically distinguish between an $I$ (1) series from an $I(0)$ series contaminated by a structural shift is therefore required. When the break point is known, Perron's test (1989) can be used. However, when the change point is unknown, Banerjee et al. (1992) test or Zivot and Andrews (1992) test can be run. Since both procedures are quite similar, we only present the results obtained by running Zivot and Andrews' procedure. This sequential test procedure consists of using a dummy variable for each possible break date. The break point corresponds to the minimum of the Student's $t$ stat obtained from the ADF procedure test. Figure 2c displays the Student's t ratio statistic for a possible break affecting the intercept as well as the trend. For both series, unit root hypothesis cannot be rejected. The minimum value of the $t$ statistics for the crude oil price series and for the gasoline price are -3.85 and -4.56 , respectively (the $5 \%$ critical value is -4.82 , see Fig. $2 \mathrm{c}$ ).

\subsection{Test for time-varying cointegration under possible break}

The standard CADF test for testing no-cointegration cannot reject the null hypothesis regardless of the selected order $m$. However, for the same reason as for the unit root tests, under structural break, tests for cointegration tend to under-reject the null of nocointegration (Gregory et al. 1996). In order to overcome this problem, we performed the FMLS-based CUSUM cointegration test proposed by Xiao and Phillips (2002). These authors extend the traditional OLS-based CUSUM test for structural change by considering the FMLS residuals and the estimated long-run variance estimate instead of the OLS residuals and the estimated error variance. Xiao and Phillips (2002) showed how this statistic can be used to test the null hypothesis of cointegration against the alternative of no-cointegration. The FMLS-based CUSUM statistics is given by:

$$
\mathrm{CS}_{T}=\max _{k=1, \ldots, T} \frac{1}{\sqrt{T \hat{\omega}_{\text {uuee }}}}\left|\sum_{t=1}^{k} \hat{u}_{t}^{+}\right|,
$$

where $\hat{u}_{t}^{+}=y_{t}^{+}-\hat{\theta}^{+}(t) x_{t}$ is the fully modified residual process. The asymptotic distribution of $\mathrm{CS}_{T}$ under the null hypothesis of (time-invariant) cointegration and critical values (without trend) are given by Xiao and Phillips (2002).

Now, under the null hypothesis of time-varying cointegration, it is straightforward to obtain the limiting distribution of $\mathrm{CS}_{T}$ using the asymptotic results of Xiao and Phillips (2002) and BM (2010). We have:

$$
\mathrm{CS}_{T} \Longrightarrow \sup _{r \in[0,1]}|W(r)|
$$


Table 4 Upper tail critical values for FMLS-based CUSUM statistic under time-varying cointegration, for $p=2$

\begin{tabular}{llllllll}
\hline & $m=1$ & $m=2$ & $m=3$ & $m=4$ & $m=5$ & $m=6$ & $m=7$ \\
\hline 0.90 & 1.99 & 2.39 & 2.78 & 3.24 & 3.57 & 3.87 & 4.23 \\
0.95 & 2.33 & 2.89 & 3.40 & 4.03 & 4.37 & 4.73 & 5.14 \\
0.975 & 2.68 & 3.41 & 3.98 & 4.74 & 5.22 & 5.61 & 6.11 \\
0.99 & 3.13 & 4.08 & 4.86 & 5.64 & 6.32 & 6.57 & 7.21 \\
\hline
\end{tabular}

Calculated from 10,000 replications

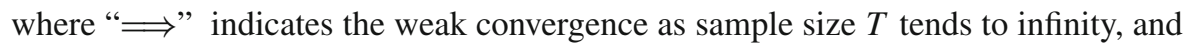
where $W(r)$ is defined as follows:

$$
W(r)=W_{1}(r)-\left(\int_{0}^{1} d W_{1}(r) S^{(m)}(r)^{\prime}\right)\left(\int_{0}^{1} S^{(m)}(r) S^{(m)}(r)^{\prime}\right)^{-1} \int_{0}^{r} S^{(m)}(r) \mathrm{d} r
$$

with

$$
\begin{aligned}
& S^{(m)}(r)^{\prime} \\
& =\left(W_{2}(r)^{\prime}, \sqrt{2} \cos (\pi r) W_{2}(r)^{\prime}, \ldots, \sqrt{2} \cos (i \pi r) W_{2}(r)^{\prime}, \ldots, \sqrt{2} \cos (m \pi r) W_{2}(r)^{\prime}\right),
\end{aligned}
$$

where $W_{1}(r)$ and $W_{2}(r)$ are 1 and $(p-1)$-dimensional standard Brownian motions that are independent of each other (see Appendix for some details). Table 4 provides the critical values (for $p=2$ and $m=(1,2, \ldots, 7)$ ) obtained using a direct simulation with a sample size 1,000 and evaluating the stochastic integrals at 10,000 points over the argument $r$. The critical values are computed as empirical quantiles from the replications. Table 4 and Fig. 3 show that these distributions get more skewed as the order $m$ increases. Note that for $m=0$, which corresponds to the time-invariant cointegration, critical values are provided in Xiao and Phillips (2002).

We ran, for $m=1$ to $m=10$, the FMLS-based CUSUM test for our single timevarying cointegrating equation. Except for $m=1$, the FMLS-based CUSUM test cannot reject the null of time-varying cointegration.

\subsection{Test for structural break under time-varying cointegration}

Combining the HQC and the significance of each Fourier coefficient to select $m$, the order $m=2$ was retained. For this polynomial order, the FMLS-based CUSUM statistic value is 2.7 (the associated critical value is 2.9 at $5 \%$ ) which lead us to cannot reject the null of cointegration. Figure 4 a compares the regressor features (i.e. the crude oil price itself (i.e. $i=0$ ) and its product with the first- and second-order Chebyshev time polynomial $(i=1,2))$. In Sect. 2.1, we showed that if the crude oil price was $I$ (1) therefore, its product with $G_{1, T}$ and $G_{2, T}$ also will be $I(1)$, which seems to be confirmed by Fig. 4a. Figure $4 \mathrm{~b}$ plots the $L M$ statistics sequence over the search grid (with $\tau_{L}=1-\tau_{U}=0.15$ ). The obtained supremum $L M$ statistic is equal to 


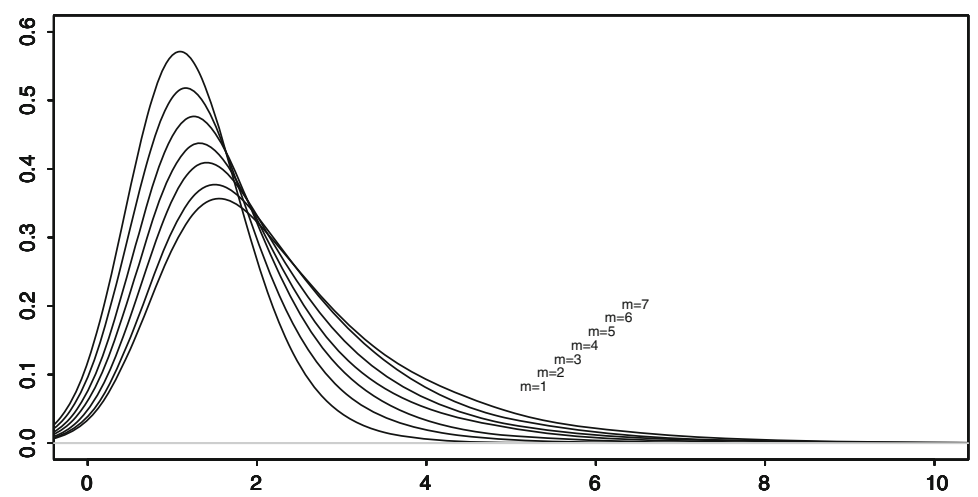

Fig. 3 Asymptotic distribution (obtained with a kernel estimator) of FMLS-based CUSUM statistics under time-varying cointegration for $p=2$. Distributions obtained from direct simulation with a sample size $T=1,000$ and 10,000 replications

102.1 which lead us to strongly reject the null hypothesis of stability of the Fourier parameters.

\subsection{Estimating the break date}

Regression (6) is obviously infeasible if the break point is unknown. A feasible approach consists of first estimate the break date, and then estimate the model by FMLS using the estimated break point. By referring to the standard econometric framework (the linear and stationary case), a natural candidate for a break date estimate is the date associated to the largest value of the used statistic test sequence. In linear regression and under homoscedasticity, this is true for the Wald test statistic since it is a monotonic transformation of the sum-of-squared residuals (SSR). Hence, the date that maximizes the Wald statistic sequence is the date that minimizes the SSR. Bai et al. (1998) provided inference for breaks in (time-invariant) cointegrating parameters. However, the authors consider a special cointegrated system where the error process $e_{t}$ in (1) is assumed to be independent of the regressors, which is not our context.

Relaxing the absence of correlation between both terms $e_{t}$ and $u_{t}$, Kurozumi and Arai (2005) investigated the consistency and the asymptotic properties of $\hat{\tau}$ estimated as follows:

$$
\hat{\tau}=\arg \inf _{\tau \in\left[\tau_{L}, \tau_{U}\right]} \operatorname{SSR}_{T}(\tau),
$$

where $\operatorname{SSR}_{T}(\tau)=T^{-1} \sum_{t=1}^{T} \hat{u}_{t \tau}^{2}$, and where $\hat{u}_{t \tau}$ are the residuals obtained, for example, by running the OLS on a feasible version of the regression (6). The author show that such a correlation does not affect the asymptotic property of $\hat{\tau}$ and that $T^{1-2 \kappa}\left(\hat{\tau}-\hat{\tau}_{o}\right)$ is $O_{p}(1)$ for $\kappa \in[0,1 / 2)$, where $\kappa$ represents the magnitude of the break. They also show that the limiting distribution of $\hat{\tau}$ is the same as the one given in Bai et al. (1998). Therefore, following Kurozumi and Arai (2005), the estimate of the break date $\left[T \tau_{o}\right]$ based on (13) is acceptable. Once the break fraction $\tau$ is estimated, an efficient esti- 

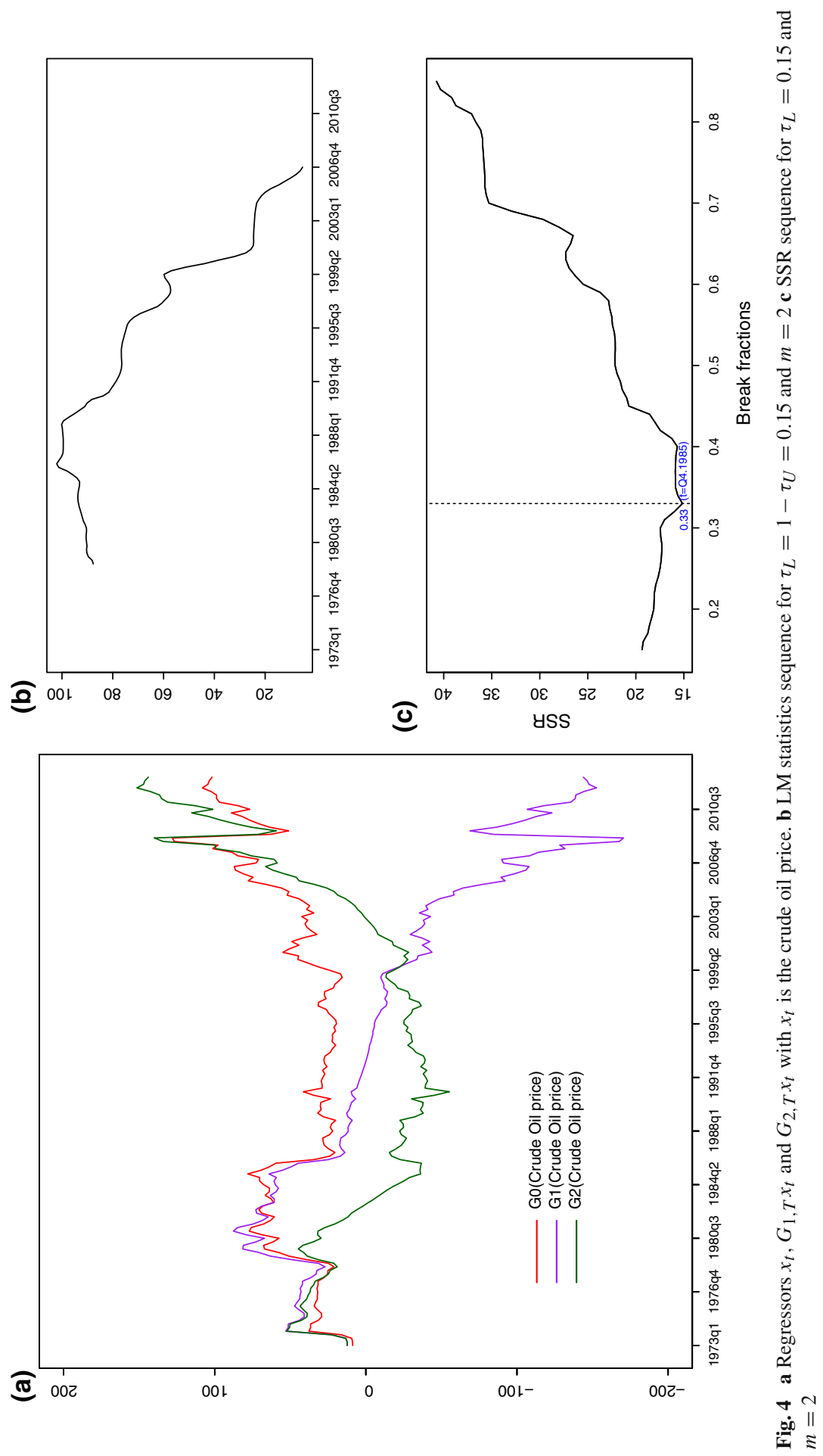
mation procedure can be performed to estimate the Fourier coefficients. Kurozumi and Arai (2005) use Park (1992)'s canonical cointegrating regression (CCR). The authors show the CCR consistency in (time-invariant) cointegrating regressions with structural change. They also point out that their results hold for the FMLS, since the only difference between these two estimators resides in the way to correct serial correlations.

For all $m=1, \ldots, 10$, the procedure leads to the same break date estimate: $\hat{\tau}=$ $0.33,[T \hat{\tau}]=Q 4.1985$, which is in line with the graphical diagnostic provided by the scatter plot being displayed in Fig. 2b. Figure 4c provides the output of (13) for $m=2$ and $\tau_{L}=0.15$.

\subsection{Model estimates}

For the estimated break point, the ADF residual-based test for no-cointegration (CADF statistic) and the FM Wald test for time-invariant cointegration hypothesis (described in sect. 2.2) were performed. Both reject the null hypothesis at $\alpha=5 \%$. The estimates are given bellow ${ }^{(a)}$ :

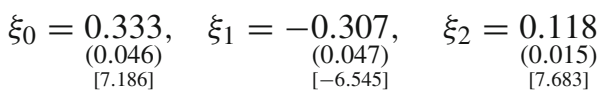

$$
\begin{aligned}
& \xi_{c, 0}=\underset{(0.046)}{-0.295}, \quad \xi_{c, 1}=\underset{\substack{(0.047) \\
[6.697]}}{0.314}, \quad \xi_{c, 2}=-\underset{(0.016)}{-0.125}
\end{aligned}
$$

CADF test: $\rho=0.522, \quad t$ stat $=-6.963^{(\mathrm{b})}$

Time-invariant cointegration test: FM Wald $=626.38^{(\mathrm{c})}$

(a) (se), [t-stat]. (b) The number of lags in the CADF regression was selected by minimizing the AIC. (Critical values for $p>3$ are given in MacKinnon (2010)). (c) The null hypothesis of time-invariant hypothesis is rejected at $5 \%$ ( $p$ value $<0.001$, according a $\chi_{(4)}^{2}$ criterion).

In order to assess the response of the retail gasoline price to the oil crude price after and before the structural change, the time-varying slope coefficients $\theta^{+}(t)$ are plotted in Fig. 5a, b with the 5 and $10 \%$ confidence bounds. Thus, the figure shows that the response of Swiss retail price to the crude oil price became suddenly stronger after the break. The response of the Swiss price of gasoline reached a level close to the one after the oil shock in 1973. Since this break date, the retail price response dynamic seems to return slowly to lower values. Hence, it is only in 2005 that the response for retail gasoline price got back to levels similar to the ones between 1980 and 1984. Since then, the retail price response has not stopped to decrease slowly and become not significant in the end of the period in 2010 (see Fig. 5c). Figure 5d plots the FM residuals for which the feature seems to be stationary as confirmed by CADF test result reported above. 

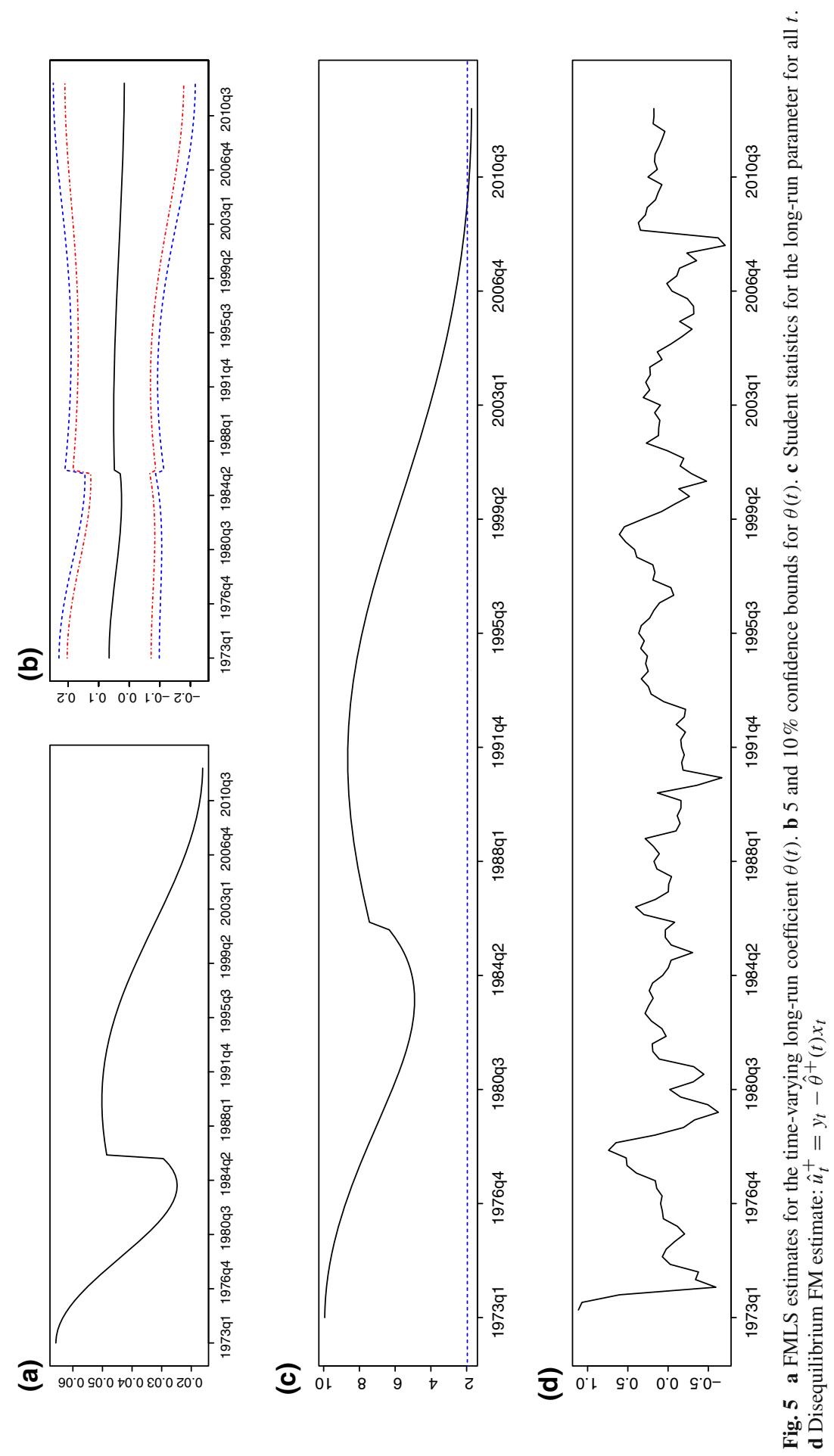


\section{Conclusion}

In this paper, we combine smooth time-varying long-run parameters and structural break in a single cointegration relationship. We outline how to exploit Hansen (1992) procedure for testing structural break in such a model. Our methodology is used to investigate the long-run relationship between the crude oil price and the retail gasoline price. We show that time-invariant parameters in the considered long-run relationship are too restrictive to validate the cointegration hypothesis. Hence, it can be worth for practitioners to test occurrence of a structural break in a smooth time-varying long-run dynamic, since the cointegration hypothesis could not be established in our empirical case without considering this specification. However, it is important to note that such a procedure can be exposed to the end-point problem, in particular if the fluctuations of the time-varying parameters exhibit strong peaks. Hence, in the search grid, if a break at $t$ is tested, the accuracy of the estimate at $t-1$ is crucial to make the right decision. Unfortunately, it is at this point that the risk of instability of the polynomial approximation is the strongest and, despite the use of a supremum statistic, the procedure can lead to an over-rejection of the parameter stability.

\section{Appendix}

The limiting distribution of $\mathrm{CS}_{T}$ under time-varying cointegration can be obtained using Lemma 2 in Bierens and Martins (2010) and Theorem 1 of Xiao and Phillips (2002).

Lemma A.1 Under the assumption of $\Delta x_{t}$ being a strictly stationary zero-mean $p$ variate process such that $\Delta x_{t}=e_{t}$ with $e_{t}$ i.i.d $(0, \Omega)$, defining $Z_{t}$ as in equation (7) and denoting $\Omega^{\frac{1}{2}}$ as the Cholesky factorization of $\Omega$, i.e. $\Omega=\Omega^{\frac{1}{2}} \Omega^{\frac{1}{2}{ }^{\prime}}$, using Lemma 2 of Bierens and Martins (2010), it comes the following limiting distributions:

$$
\begin{aligned}
& \frac{1}{T} \sum_{t=1}^{T} Z_{t}^{\prime} e_{t} \Longrightarrow \Omega^{\frac{1}{2}} \int_{0}^{1} S^{(m) \prime}(r) d W_{2}(r), \\
& \frac{1}{T^{2}} \sum_{t=1}^{T} Z_{t}^{\prime} Z_{t} \Longrightarrow \Omega^{\frac{1}{2}}\left(\int_{0}^{1} S^{(m) \prime}(r) S^{(m)}(r) d r\right) \Omega^{\frac{1}{2}},
\end{aligned}
$$

where $S^{(m)}(r)$ is defined as:

$$
S^{(m)}(r)^{\prime}=\left(W_{2}(r)^{\prime}, \sqrt{2} \cos (\pi r) W_{2}(r)^{\prime}, \ldots, \sqrt{2} \cos (m \pi r) W_{2}(r)^{\prime}\right)
$$

where $W_{2}(r)$ is a $(p-1)$ vector Brownian motion.

Note that the above results are immediate if $e_{t}$ is serially correlated such as $e_{t}=$ $\Phi(L) v_{t}=\sum_{s=0}^{\infty} \Phi_{s} e_{t-s}$, and we assume that $\sum_{s=0}^{\infty} s\left|\phi_{i j}^{(s)}\right|<\infty$ for each $i, j=1, \ldots, n$ for $\phi_{i j}^{(s)}$, the row $i$, column $j$ element of $\Phi_{s}$ and that $\left(v_{t}\right)$ is an i.i.d. sequence with mean 
0 , variance $\Omega_{v}$ with the factorization $\Omega_{v}=\Omega_{v}^{\frac{1}{2}} \Omega_{v}^{\frac{1}{2}}$, finite fourth moments. We have in this case:

$$
\frac{1}{T} \sum_{t=1}^{T} Z_{t}^{\prime} e_{t} \Longrightarrow \Lambda^{\frac{1}{2}}\left(\int_{0}^{1} S^{(m) \prime}(r) d W_{2}(r)\right) \Lambda^{\frac{1}{2}{ }^{\prime}}+\sum_{k=1}^{\infty} \Omega_{k}
$$

and

$$
\frac{1}{T^{2}} \sum_{t=1}^{T} Z_{t}^{\prime} Z_{t} \Longrightarrow \Lambda^{\frac{1}{2}}\left(\int_{0}^{1} S^{(m) \prime}(r) S^{(m)}(r) d r\right) \Lambda^{\frac{1}{2}{ }^{\prime}},
$$

where $\Omega_{s}=\mathbb{E}\left(e_{t} e_{t-s}^{\prime}\right)=\sum_{k=0}^{\infty} \Phi_{s+k} \Omega_{v} \Phi_{k}^{\prime}$ for $s=0,1,2, \ldots$, and where $\Lambda^{\frac{1}{2}}=$ $\Phi(1) \Omega_{v}^{\frac{1}{2}}$ with $\Phi(1)$ nonsingular.

Using the previous results and following Phillips and Hansen (1990) and Phillips (1995) to establish the limiting distribution of $1 / \sqrt{T}\left|\sum_{t=1}^{[T r]} \hat{u}_{t}^{+}\right|$, with $r \in[0,1]$, the convergence (12) can be stated, which is similar to the standard time-invariant case presented in Theorem 1 of Xiao and Phillips (2002).

\section{References}

Bai J, Lumsdaine RL, Stock JH (1998) Testing for and dating common breaks in multivariate time series. Rev Econ Stud 65:395-432

Balke HS, Fomby TB (1997) Threshold cointegration. Int Econ Rev 38:627-645

Banerjee A, Lumsdaine RL, Stock JH (1992) Recursive and sequential tests of the unit-root and trend-break hypotheses: theory and international evidence. J Bus Econ Stat 10:271-287

Bierens HJ (1997) Testing the unit root with drift hypothesis against nonlinear trend stationarity, with an application to the US price level and interest rate. J Econom 81:29-64

Bierens HJ, Martins LF (2010) Time-varying cointegration. Econom Theory 26:1453-1490

Enders W, Siklos PL (2001) Cointegration and threshold adjustment. J Bus Econ Stat 19:166-176

Gonzalo J, Pitarakis PY (2006) Threshold effects in multivariate error correction models. In: Mill TC, Patterson K (eds) Palgrave handbook of econometrics, vol 1. Palgrave Macmillan, Hampshire, pp 578-609

Gregory AW, Hansen BE (1996) Residual-based test for cointegration in models with regime shifts. J Econom 70:99-126

Gregory AW, Nason JM, Watt D (1996) Testing for structural breaks in cointegrated relationships. J Econom 71:321-341

Hansen BE (1992) Tests for parameter instability in regressions with I(1) processes. J Bus Econ Stat 10:321-335

Hansen BE, Seo B (2002) Testing for two-regime threshold cointegration in vector error-correction models. J Econom 110:293-318

Krishnakumar J, Neto D (2011) Testing the inaction corridor in a three-regime threshold error correction model: an application to a Buffer-Stock model for U.S. money demand. Econ Notes 40:29-43

Krishnakumar J, Neto D (2012) Testing uncovered interest rate parity and term structure using three-regime threshold unit root VECM: an application to the Swiss interest rates islet. Oxf Bull Econ Stat 74:180202

MacKinnon JG (2010) Critical values for cointegration tests. Queen's Economics Department Working Paper, 1227 
Neto D (2012) Testing and estimating time-varying elasticities of Swiss gasoline demand. Energy Econ 34:1755-1762

Park JY, Phillips PCB (1988) Statistical inference in regressions with integrated processes: part I. Econom Theory 4:468-497

Park JY (1992) Canonical cointegrating regression. Econometrica 60:119-143

Park JY, Hahn SB (1999) Cointegrating regressions with time varying coefficients. Econom Theory 15:664703

Park SY, Zaho G (2010) An estimation of U.S. gasoline demand: a smooth time-varying cointegration approach. Energy Econ 32:110-120

Perron P (1989) The great crash, the oil price shock, and the unit root hypothesis. Econometrica 57:13611401

Phillips PCB, Hansen BE (1990) Statistical inference in instrumental variables regression with I(1) processes. Rev Econ Stud 57:99-125

Phillips PCB (1995) Fully modified least squares and vector autoregression. Econometrica 63:1023-1078

Kurozumi E, Arai Y (2005) Efficient estimation and inference in cointegrating regressions with structural change. J Time Ser Anal 28:545-575

Seo M (2006) Bootstrap testing for the null of no cointegration in a threshold vector error correction model. J Econom 134:129-150

Xiao Z, Phillips PCB (2002) A CUSUM test for cointegration using regression residuals. J Econom 108:4361

Xiao Z (2009) Functional-coefficient cointegration models. J Econ 152:81-92

Zivot E, Andrews DWK (1992) Further evidence on the great crash, the oil-price shock, and the unit-root hypothesis. J Bus Econ Stat 10:251-270 